\title{
A Comparison between a Manual and Automatic Method to Characterize Red Blood Cell Trajectories
}

\author{
Diana Pinho*, Fernando Gayubo ${ }^{\S}$, Ana I. Pereira*,£ and Rui Lima**** \\ * Polytechnic Institute of Bragança, Portugal \\ ${ }^{\S}$ Fundación CARTIF, Valladolid, Spain \\ ${ }^{£}$ Algoritmi, University of Minho, Portugal \\ ** CEFT, FEUP, Porto University, Portugal
}

\begin{abstract}
The current study proposes an automatic method for the segmentation and tracking of red blood cells (RBCs) flowing through a $100 \mu \mathrm{m}$ glass capillary. The measurements obtained with the proposed automatic method are compared with a manual tracking method using nonlinear optimization techniques.
\end{abstract}

Keywords: Automatic Method. Red Blood Cells. Nonlinear Optimization.

PACS: $02.60 . P n$

\section{INTRODUCTION}

Blood flow in microcirculation is crucial for the normal function of tissues and organs. Detailed study of blood cells flowing in microvessels and microchannels is essential to provide a better understanding on the blood rheological properties and disorders in microcirculation [1-5]. Image analysis plays an extremely important role to obtain information about the blood rheology. However, most of the past blood flow experimental data have been performed manually [1-3]. Although manual methods can be highly reliable, these methods are relatively time consuming and can also introduce user errors into the data. As a result, it is crucial to develop image analysis methods able to get the data automatically. The present paper presents an approach able to track the RBCs trajectories automatically. Nonlinear optimization techniques were used to compare automatic and manual methods.

\section{MATERIALS AND METHODS}

\section{Experimental Set-up}

The confocal system used in this study consists of an inverted microscope (IX71; Olympus) combined with a confocal scanning unit (CSU22; Yokogawa), a diode-pumped solid-state (DPSS) laser (Laser Quantum) with an excitation wavelength of $532 \mathrm{~nm}$ and a high-speed camera (Phantom v7.1; Vision Research). The laser beam was illuminated from below the microscope stage through a dry $40 \times$ objective lens. The glass capillary was placed on the stage of the inverted microscope and by using a syringe pump (KD Scientific) a pressure-driven flow was kept constant $(\operatorname{Re} \sim 0.008)$. More detailed information about this system can be found elsewhere [1].

The confocal images were captured around the middle of the capillary with a resolution of $640 \times 480$ pixel at a rate of 100 frames/s. Two image analyses methods were used in this study: manual method (MM) and automatic method (AM).

Numerical Analysis and Applied Mathematics ICNAAM 2011

AIP Conf. Proc. 1389, 767-770 (2011); doi: 10.1063/1.3636845

(C) 2011 American Institute of Physics 978-0-7354-0956-9/\$30.00 
A manual tracking plugin (MTrackJ) [6] of an image analysis software (Image J, NIH) [7] was used to track individual RBC. By using MTrackJ plugin, the bright centroid of the selected RBC was automatically computed through successive images for an interval of time of $10 \mathrm{~ms}$. After obtaining $x$ and $y$ positions, the data were exported for the determination of each individual RBC trajectory.

\section{Automatic Method (AM)}

All frames were loaded and pre-processed using Matlab [8]. The region of interest was then cropped from the images with the function imcrop. The median function, medfilt2, with one mask $5 \times 5$ pixel, was applied to eliminate most of the noise and to enhance the flowing object. In Fig. 1 we can see the result of these processing steps. In the next step, the images are subject to a segmentation filter, Sobel. With this segmentation it is possible to separate $\mathrm{RBC}$ from the background, i.e. differentiate the area of interest (the RBCs) from the not-interest area (background image). This is possible using a threshold method, where a definition of one or more values of separation is enough to divide the image into one or more regions. The function iterative threshold was applied for the sequence of all the images.
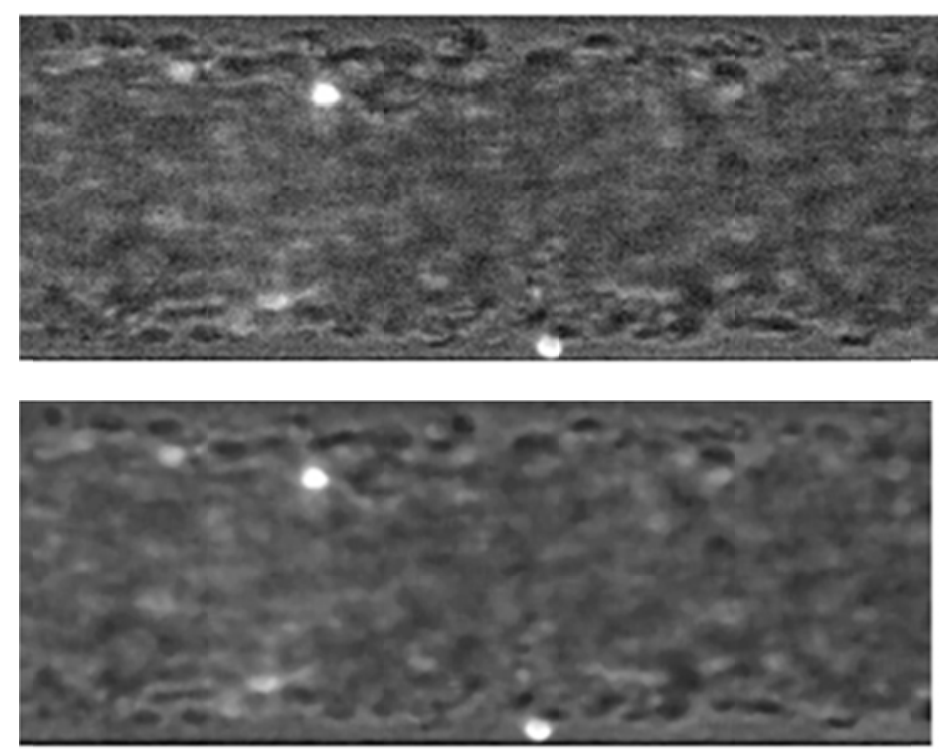

FIGURE 1. The region of interest (above) and the image filtered by using the median function medfilt2.

The objects are defined with the Sobel filter (see Fig. 2), which shows only the edge of the objects. The Sobel computes an approximation of the gradient of the image intensity. At each pixel point in the image, the result of the Sobel operator is either the corresponding gradient vector or the norm of this vector. More detailed information about this automatic method can be found elsewhere [9].

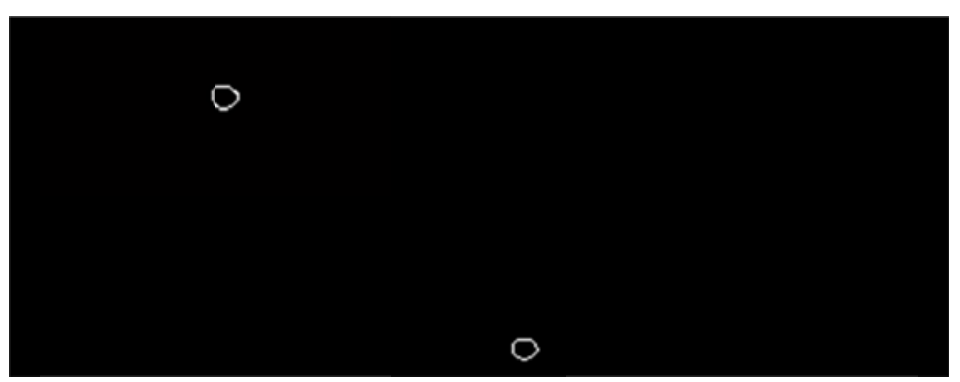

FIGURE 2. Result of the iterative threshold method and the filter Sobel. 


\section{RESULTS AND DISCUSSION}

After obtaining series of $x$ and $y$ positions for both methods, data were exported for the determination of each individual $\mathrm{RBC}$ trajectory and to analyze the best mathematical function that approximates to the RBCs experimental flow behavior.

In this study it was measured and analyzed twenty five red blood cells at a temperature of $25^{\circ} \mathrm{C}$. For each cell $i$, by using the MTrackJ plugin (MM) form ImageJ and the propose method (AM), we have obtained $\left\{\left(x_{i}, y_{j}\right), j=\right.$ $\left.1, \ldots, k_{i}\right\}$ data. To compare the consistency of both methods we decided to determine the better approximation for the data by using a nonlinear optimization [10]. Hence, we consider three different functions [5] (polynomials, sum of trigonometric functions and a sum of exponential functions) defined as:

$$
f_{1}(x, p)=\sum_{i=0}^{9} p_{i} x_{i} ; \quad f_{2}(x, a, b)=a_{0}+\sum_{i=1}^{8} a_{i} \cos (\mathrm{ix})+b_{i} \sin (i x) ; f_{3}(x, c, d, g)=\sum_{i=1}^{8} g_{i} e^{\left(-\left(\frac{\left(x-c_{i}\right)}{d_{i}}\right)^{2}\right)} .
$$

where $p \in I R^{10}, a \in I R^{9}, b, c, d, g \in I R^{8}$ are the function parameters and the vector $x \in I R^{k i}$, where $i$ represents the cell number. To identify the functions parameters it was used the tool cftool present in Curve Fitting Toolbox from Matlab [11].

The error of nonlinear least squares approximation of the selected RBCs are listed in the Table 1, where RBC refers to the red blood cell number, $F_{i}=\sum_{j=i}^{k i}\left(y_{i}-f_{i}\left(x_{i}\right)\right)^{2}$ is the nonlinear least squares approximation error of the function $f_{i}(x,$.$) , with i=1,2,3$, Av refers the error average, and $\mathbf{s}$ corresponds to the standard deviation of the errors.

Table 1 - Errors of nonlinear least squares approximation of the selected RBCs for both methods.

\begin{tabular}{lccccccc}
\hline & \multicolumn{3}{l}{ Manual Method (MM) } & & & \multicolumn{2}{c}{ Automatic Method (AM) } \\
\hline RBC & $F_{1}$ & $F_{2}$ & $F_{3}$ & $\mathbf{R B C}$ & $F_{1}$ & $F_{2}$ & $F_{3}$ \\
\hline $\mathbf{1}$ & 0.5802 & 0.1998 & 0.4603 & $\mathbf{1}$ & 0.8738 & 0.2871 & 0.4787 \\
$\mathbf{2}$ & 1.2620 & 0.4709 & 0.5414 & $\mathbf{2}$ & 0.3919 & 0.3039 & 0.2493 \\
$\mathbf{3}$ & 0.5802 & 0.1717 & 0.4328 & $\mathbf{3}$ & 0.8912 & 0.6923 & 0.7849 \\
$\mathbf{4}$ & 0.9940 & 0.4970 & 1.0120 & $\mathbf{4}$ & 0.4644 & 0.1990 & 0.2304 \\
$\mathbf{5}$ & 1.3050 & 0.8490 & 1.2900 & $\mathbf{5}$ & 0.7941 & 0.5489 & 0.5799 \\
$\mathbf{6}$ & 0.1937 & 0.05658 & 0.1041 & $\mathbf{6}$ & 0.3110 & 0.1946 & 0.1991 \\
$\mathbf{7}$ & 0.1587 & 0.0754 & 0.0481 & $\mathbf{7}$ & 0.2538 & 0.1126 & 0.1952 \\
$\mathbf{8}$ & 0.4979 & 0.2534 & 0.3243 & $\mathbf{8}$ & 0.1833 & 0.1546 & 0.4099 \\
$\mathbf{9}$ & 0.5313 & 0.2296 & 0.2592 & $\mathbf{9}$ & 0.5139 & 0.4374 & 0.3243 \\
$\mathbf{1 0}$ & 0.8231 & 0.5358 & 0.5611 & $\mathbf{1 0}$ & 0.6409 & 0.3136 & 0.5406 \\
$\mathbf{1 1}$ & 0.1833 & 0.0834 & 0.1454 & $\mathbf{1 1}$ & 0.2259 & 0.1382 & 0.1071 \\
$\mathbf{1 2}$ & 0.4453 & 0.2673 & 0.2395 & $\mathbf{1 2}$ & 0.9160 & 0.6922 & 0.7696 \\
$\mathbf{1 3}$ & 0.6081 & 0.2685 & 0.5399 & $\mathbf{1 3}$ & 0.4071 & 0.2730 & 0.9612 \\
$\mathbf{1 4}$ & 1.6060 & 0.6657 & 1.0260 & $\mathbf{1 4}$ & 0.2392 & 0.1920 & 0.1821 \\
$\mathbf{1 5}$ & 0.7841 & 0.4323 & 1.4060 & $\mathbf{1 5}$ & 3.0220 & 2.0240 & 2.5100 \\
$\mathbf{1 6}$ & 0.6095 & 0.2851 & 1.0860 & $\mathbf{1 6}$ & 5.7790 & 4.8450 & 4.7570 \\
$\mathbf{1 7}$ & 1.0890 & 0.6595 & 1.6690 & $\mathbf{1 7}$ & 1.7780 & 1.6890 & 1.6960 \\
$\mathbf{1 8}$ & 5.2040 & 1.9490 & 11.7300 & $\mathbf{1 8}$ & 1.3980 & 0.9345 & 2.0040 \\
$\mathbf{1 9}$ & 0.3997 & 0.3997 & 0.2499 & $\mathbf{1 9}$ & 0.6008 & 0.3624 & 0.4362 \\
$\mathbf{2 0}$ & 0.5611 & 0.3689 & 0.3277 & $\mathbf{2 0}$ & 0.9570 & 0.3418 & 0.3859 \\
$\mathbf{2 1}$ & 0.1833 & 0.0965 & 0.1396 & $\mathbf{2 1}$ & 0.7467 & 0.3855 & 0.8311 \\
$\mathbf{2 2}$ & 0.9831 & 0.5297 & 0.2943 & $\mathbf{2 2}$ & 1.2090 & 0.6394 & 1.0780 \\
$\mathbf{2 3}$ & 1.1230 & 0.4937 & 0.4924 & $\mathbf{2 3}$ & 0.5624 & 0.2787 & 0.2345 \\
$\mathbf{2 4}$ & 2.9160 & 1.5330 & 1.8520 & $\mathbf{2 4}$ & 6.1240 & 2.5090 & 3.3190 \\
$\mathbf{2 5}$ & 4.9850 & 1.3170 & 2.0980 & $\mathbf{2 5}$ & 2.4780 & 0.9938 & 1.3230 \\
\hline $\mathbf{A v}$ & 1.144 & $\mathbf{0 . 5 0 8 0}$ & 1.1330 & $\mathbf{A v}$ & 1.2700 & $\mathbf{0 . 7 8 2 0}$ & 0.9360 \\
\hline $\mathbf{s}$ & 1.3230 & $\mathbf{0 . 4 6 7 0}$ & 2.2830 & $\mathbf{s}$ & 1.5690 & $\mathbf{1 . 0 4 4 0}$ & 1.1310 \\
\hline
\end{tabular}

The results, from Table 1 , indicate that the function $f_{2}(x, a, b)$ (sum of trigonometric functions) was the best approximation, to the motion of the RBCs flowing in microchannels, for both methods. This is confirmed by the 
value of the error average. Another important aspect is the fact that the standard deviation of the errors is small when we use the function $f_{2}$.

\section{CONCLUSIONS}

The present study indicates that the data obtained from the proposed automatic method (AM) is equivalent to data obtained from the manual method (MM) as the function that best approaches is the same, i.e., the function $f_{2}$ (sum of trigonometric functions). Hence, the proposed automatic method is a promising way to track blood cells flowing in microchannels.

\section{ACKNOWLEDGMENTS}

The authors acknowledge the financial support provided by: PTDC/SAU-BEB/108728/2008, PTDC/SAUBEB/105650/2008 and PTDC/EME-MFE/099109/2008 from the FCT (Science and Technology Foundation) and COMPETE, Portugal.

\section{REFERENCES}

1. R. Lima, T. Ishikawa, Y. Imai, M. Takeda, S. Wada, and T. Yamaguchi, Measurement of individual red blood cell motions under high hematocrit conditions using a confocal micro-PTV system. Annals of Biomedical Engineering. 37, 1546-59, 2009.

2. H. Fujiwara, T. Ishikawa, R. Lima, et al. Red blood cell motions in high-hematocrit blood flowing through a stenosed microchannel. Journal of Biomechanics 42, 838-843, 2009.

3. Y. Suzuki, N. Tateishi, M. Soutani and N. Maeda, Deformation of erythrocytes in microvessels and glass capillaries: effects of erythrocyte deformability. Microcirculation 3, 49-57, 1996.

4. A. Pries, T. Secomb, et al, Resistance to blood flow in microvessels in vivo. Circulation Research 75, 904-915, 1994.

5. D. Pinho, et al., Red blood cells motion in a glass microchannel, Numerical Analysis and Applied Mathematics, Vol. 1281: 963-966, 2010.

6. E. Meijering, I. Smal and G. Danuser, Tracking in molecular bioimaging, IEEE Signal Process. Mag. 23: 46-53, 2006.

7. M. Abramoff, P. Magelhaes and S. Ram, Image processing with image J, Biophotonics Int. 11: 36-42, 2004.

8. S. L. Eddins, R. C. Gonzalez, R. E. Woods, Digital Image Processing Using Matlab, 2002.

9. D. Pinho, Determination and characterization of red blood cells trajectories: a semi-automatic method, Master in Biomedical Technology, Polytechnic Institute of Bragança, Portugal, 2011 (in portuguese).

10. J. Nocedal and S. Wright, Numerical Optimization. Springer Series in Operations Research, Springer, New York, 1999.

11. MathWorks, Optimization Toolbox User's Guide, 2010. 